\title{
Research on Home Appliance Control System based on Brain-Computer Interface
}

\author{
Xiaolong Jia ${ }^{1,}$, Liqing Geng ${ }^{2, ~ b}$, Huiya Liu \\ ${ }^{1}$ Tianjin Key Laboratory of Information Sensing and Intelligent Control, Tianjin University of \\ Technology and Education, Tianjin 300222, China; \\ 2 Tianjin Key Laboratory of Information Sensing and Intelligent Control, Tianjin University of \\ Technology and Education, Tianjin 300222, China. \\ asandy860789com@163.com, bgenglq2004@126.com
}

Keywords: steady-state visual evoked potential (SSVEP), brain-computer interface, LabVIEW

\begin{abstract}
This system provides steady-state visual evoked frequency by using different-frequency LED, constructs the human-computer interface with LabVIEW platform and outputs related control command through serial port, thus realizing home appliance control. All 8 experimental subjects could accomplish home appliance control experiments successively, showing a mean accuracy of $80 \%$ and the highest accuracy of $90 \%$. This system used SSVEP in intelligent home appliance control successfully and achieved effective intelligent control of home appliances. It laid foundations for future development of more complex intelligent home appliance system based on brain-computer interface.
\end{abstract}

\section{Introduction}

BCI (brain-computer interface) refers to the direct communication and control channel built between human brain and computer or other electronic equipment. It is independent from normal physiological output channel of brain (peripheral nervous system and muscular tissue). One important use of BCI is to provide communication and control ways for physical disabled people with external environment. It enables them to control external equipment by brain potentials in special environment or when the traditional control way is inconvenient. Visual evoked potentials (VEP) is electrical responses generated upon visual stimulation of occipital region of cerebral cortex. It could represent potential changes caused when stimulation at retina is transmitted to the occipital cortex through visual pathway. VEP could be divided into SSVEP (Steady-State Visual Evoked Potentials) and transient visual evoked potentials according to different stimulation signal. The stimulus frequency of SSVEP is generally larger than $6 \mathrm{~Hz}$ and the stimulus frequency of transient visual evoked potentials is smaller than $4 \mathrm{~Hz}$. The biggest difference between SSVEP and transient visual evoked potentials is that responses in transient visual evoked potentials caused by stimulus won't overlap in time, but those in SSVEP overlap with each other. Since SSVEP has advantages of high information transmission rate, short training time and easy feature attraction, it is a common input signal of man-machine interface.

This paper developed an intelligent home appliance system based on SSVEP. It generated visual stimulus frequency by LED and built a man-machine interface by using the LabVIEW. Different frequencies were induced by asking experimental subjects to see different-frequency LED lamp, thus enabling to control different functions of home appliances in the home system.

\section{System design and experimental process}

This system includes three functional modules, namely, brain potentials collection, signal processing and home appliance control. The system structure is shown in Fig.1. 


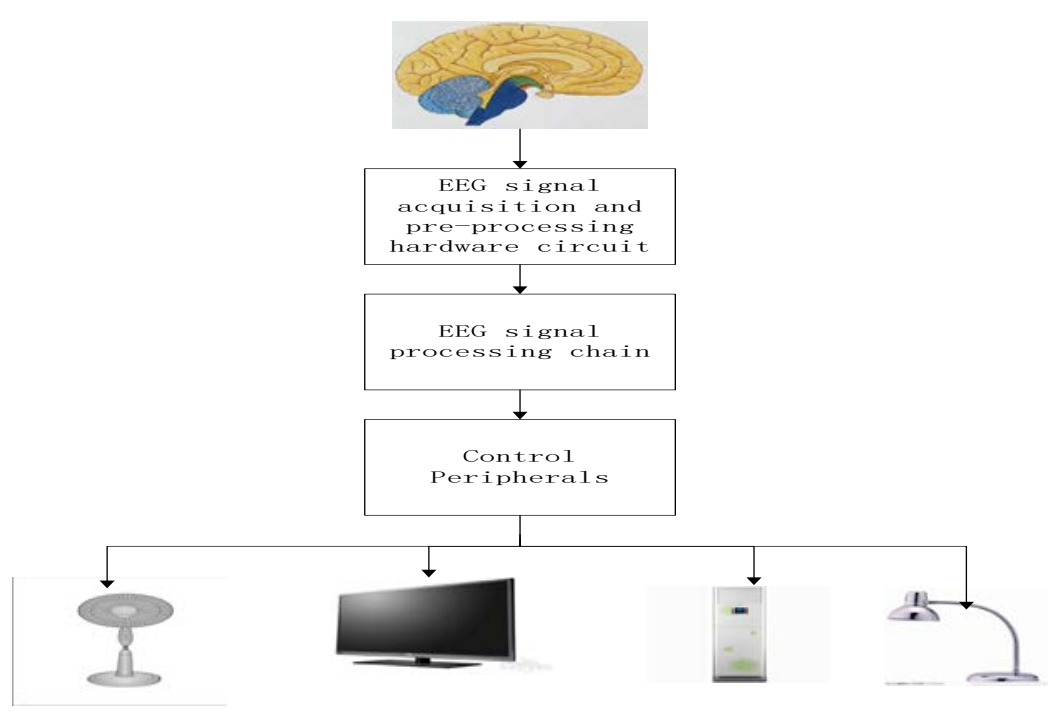

Fig.1 System structure

System stimulator applies several LED lamps of different frequencies and builds the man-machine interface by using the LabVIEW platform. Experimental subjects makes responses related with stimulus frequency by staring at LED stimulator. Associated data will be collected and transmitted by brain potentials collector into computer. The signal will be processed and its mode will be identified by the software program established by LabVIEW platform. When input data response and presetting response match with each other, the indicator light on the board in front of LabVIEW will be on, which reminds experimental subjects the current operation is successful and could go to next operation.

\section{Function Module Design}

Brain Potentials Collection. Brain potential collector used the NT9200 electroencephalograph of China Sciences Group. During the experiment, sampling rate was set $200 \mathrm{~Hz}$ and electroencephalogram signal was collected through the O1, C3 and C3. C3 and C4 were used as reference electrode. The collected data were converted into digital value through $\mathrm{A} / \mathrm{D}$ conversion and then sent into computer for further processing.

Signal Processing. Signal processing mainly includes three steps: signal preprocessing, feature extraction and pattern recognition.

Since electroencephalogram signal is very weak and external noise exist during collection, the first thing in signal preprocessing is to make band-pass filtering of $\mathrm{A} / \mathrm{D}$ converted electroencephalogram data. An ellipse digital band-pass filter was designed. The band-pass range is $10 \mathrm{~Hz} 20 \mathrm{~Hz}$ and the ripple factor within band-pass is smaller than $0.5 \mathrm{~dB}$. The attenuation coefficient within the stop-band is larger than $50 \mathrm{~dB}$.

Feature Extraction. Actually, signal processing is to analyze frequency of the collected electroencephalogram signal and extract features. The system uses Fast Fourier Transform (FFT) as the core of algorithm. FFT mainly analyzes frequency of preprocessed electroencephalogram signal to get the frequency spectral peaks which are convenient for feature extraction. Based on characteristics of Fourier coefficient, some items in the discrete Fourier transform (DFT) could be combined or long sequence could be divided into short ones. This reduces calculation workload of DFT significantly. The basic algorithm of FFT includes time-based selection and frequency-based selection. This paper mainly applied basic-2 FFT algorithm using time-based selection.

If signal $x(n)$ has $N=2^{l}$ data points and $l$ is integer. The signal is divided into two parts according to odd and even of $\mathrm{n}$ :

$$
\begin{aligned}
& x(2 q)=x_{1}(q) \\
& x(2 q+1)=x_{2}(q)
\end{aligned}
$$


Then DFT of $x(n)$ is :

$X(k)=\sum_{q=0}^{\frac{N}{2}-1} x_{1}(q)\left(W_{N}^{2}\right)^{q k}+W_{N}^{k} \sum_{q=0}^{\frac{N}{2}-1} x_{2}(q)\left(W_{N}^{2}\right)^{q k}$

The attenuation coefficient within the stop-band is larger than $50 \mathrm{~dB}$. According to the reducibility of FFT,

$X(k)=\sum_{q=0}^{\frac{N}{2}-1} x_{1}(q) W_{N / 2}^{q k}+W_{N}^{k} \sum_{q=0}^{\frac{N}{2}-1} x_{2}(q) W_{N / 2}^{q k}, k=0,1, \cdots, \frac{N}{2}-1$

At this moment, it calculate FFT at N/2 data points of $x(n)$. The FFT of another N/2 data points can be calculated from periodicity of DFT:

$X\left(k+\frac{N}{2}\right)=\sum_{q=0}^{\frac{N}{2}-1} x_{1}(q) W_{N / 2}^{q k}-W_{N}^{k} \sum_{q=0}^{\frac{N}{2}-1} x_{2}(q) W_{N / 2}^{q k}, k=0,1, \cdots, \frac{N}{2}-1$

With this method, DFT of all data points within the range of $0 \sim \mathrm{N}-1$ could be calculated if DFT of odd items and even items of all data points from $0 \sim \mathrm{N} / 201$ is known. This reduces calculation workload effectively.

Since electroencephalogram signal is very weak and external noise exist during collection, the first thing in signal preprocessing is to make band-pass filtering of A/D converted electroencephalogram data. An ellipse digital band-pass filter was designed. The band-pass range is $10 \mathrm{~Hz} \sim 20 \mathrm{~Hz}$ and the ripple factor within band-pass is smaller than $0.5 \mathrm{~dB}$. The attenuation coefficient within the stop-band is larger than $50 \mathrm{~dB}$. Since brain also will make responses related with stimulus frequency under no SSVEP, this makes it impossible to determine whether such responses are generated by experimental subjects positively or produced by the SSVEP. However, response generated by experimental subjects generally has smaller amplitude compared to that produced by SSVEP. Hence, they could be distinguished by setting a threshold value. In pattern recognition, auto power spectrum of above mentioned data was compared with the preset threshold to determine whether there's command and what command it is.

Home Appliance Control. The home appliance control modulus used multifunction remote control to verify response control of external equipments. Based on previously processed and analyzed data, whether there's command and what command it is are judged through data processing pattern recognition. Different functions of home appliances are controlled through serial ports. Home appliances in this system include TV, air conditioner, electric fan and illuminating lamps. They could be controlled by the system, such as TV channel switching, volume adjustment, speed adjustment of electric fan, etc.

\section{Experimental Design}

Experimental process is:

(1) At the beginning, experimental subjects choose the home appliance that want to control and then star at the corresponding LED to induce response. The front board will turn to the desired home appliance control interface from the full-view state.

(2) Experimental subjects could either repeat Step (1) to control functions of home appliances or return to the full-view interface by staring and withdraw from LED to choose new home appliance that want to control.

(3) The final task is to control functions of all home appliances one by one. 
Table 1 Experimental Result Statistics

\begin{tabular}{cccc}
\hline Tester & Correct Responses & Average time & Accuracy \\
\hline A & 8 & $3.3 \mathrm{~s}$ & $80 \%$ \\
B & 9 & $3.1 \mathrm{~s}$ & $90 \%$ \\
C & 9 & $3.0 \mathrm{~s}$ & $90 \%$ \\
D & 7 & $4.3 \mathrm{~s}$ & $70 \%$ \\
E & 8 & $3.5 \mathrm{~s}$ & $80 \%$ \\
F & 8 & $3.7 \mathrm{~s}$ & $80 \%$ \\
G & 7 & $3.6 \mathrm{~s}$ & $70 \%$ \\
H & 8 & $3.2 \mathrm{~s}$ & $80 \%$ \\
Mean & 8 & $3.4625 \mathrm{~s}$ & $80 \%$ \\
\hline
\end{tabular}

\section{Summary}

This system uses SSVEP as home appliance control signal, thus applying brain-computer interface into intelligent home appliance construction. This is greatly helpful to physical disabled people or the aged inconvenient in action. Experimental results show that the mean accuracy reaches $80 \%$ and the maximum accuracy reaches as high as $90 \%$, confirming the feasibility of SSVEP in intelligent home appliance system. Currently, many experiments related with BCI are still in experimental stage and there's still a long way to go to commercialize BCI products. But BCI has considerable potentials in many fields. In this paper, intelligent home appliance control is realized through SSVEP. Experimental results demonstrate that the system is stable and reliable. This is one trial for further development of intelligent home appliance system.

\section{Acknowledgments}

Fund program: Natural Science Foundation of China (61178081). Natural Science Foundation of Tianjin (14jczdjc3630). Beforehand research project for schools (jk14-13).

\section{References}

[1] Wolpaw J R, Birbaumer N, Heetderks W J. Brain-computer interface technology: a review of the first international meeting. IEEE Transactions on Rehabilitation Engineering, 2000 ; 8 (2) : 164-173

[2] Ming Cheng, ShangkaiGao, Lin Zhang. Brain-computer interface based on EEG signals[J]. Biomedical Engineering, 2009,19 (2) : 113-118

[3] Dong Ming. Progress on event related potential from sensory stimulation for brain computer interface[J].Journal of electronic measurement and instrument, 2009,23 (6) : 1-6.

[4] Feng Zhang, GuanghuaXu, Jun Xie. Review of steady state visually evoked potential[J]. Chinese Journal of scientific instrument, 2010,31 (8) : 156-165.

[5] ShangkaiGao, Comments on Recent Progress and Challenges in the Study of Brain-Computer Interface, 2007,26 (6) : 801-804. 